\title{
263. NOTA SINTAXONÓMICA SOBRE SARCOCORNIETUM PERENNIS EN CUBA E ISLAS CANARIAS
}

\author{
Antonio GALÁN DE MERA ${ }^{1 *}$, Antonio GARCÍA-FUENTES ${ }^{2}$ \\ y Eddy MARTÍNEZ-QUESADA ${ }^{3}$
}

Recibido el 19 de febrero de 2015, aceptado para su publicación el 4 de mayo de 2015

A syntaxonomic note about Sarcocornietum perennis in Cuba and Canary Islands

Palabras clave. Fitosociología, Sintaxonomía, Sarcocornia, Cuba, Canarias.

Key words. Phytosociology, Syntaxonomy, Sarcocornia, Cuba, Canary Islands.

Son ya varios los trabajos fitosociológicos que incluyen comunidades sobre suelos salinos litorales tanto en la región del Caribe (Borhidi et al., 1983; Borhidi, 1996; Martínez-Quesada 2014) como en Centroamérica (Peinado et al., 1995) y América del Sur (Galán de Mera et al., 2009), lo que nos lleva a reconsiderar algunos aspectos nomenclaturales de acuerdo con el Código Internacional de Nomenclatura Fitosociológica (Weber et al., 2000).

En uno de los primeros trabajos fitosociológicos del norte de la isla de Cuba, tras la aproximación americana de Knapp (1964), Samek (1973) describió la asociación Sarcocornietum perennis nom. mut. hoc loco [Salicornietum perennis Samek in Acad. Ci. Cuba, ser. For. 18: 46, 68 (1973); lectotypus in Borhidi (1996): 526, inv. 6, tab. 108, Alamar, Cuba] en áreas con suelos arcillosos inundados periódicamente con agua del mar y en contacto con los manglares. Esta asociación está válidamente descrita (art. 5 y 22) y citada posteriormente (Borhidi, 1996), lo que condiciona el homónimo posterior de las islas Canarias.

La "check list" sintaxonómica de España y Portugal (Rivas-Martínez et al., 2001) incluye la asociación Sarcocornietum perennis Fernández $\&$ Santos 1983. Sus autores la describieron con inventarios de los niveles inferiores del litoral canario de Lanzarote, Fuerteventura y Lobos, contactando con las comunidades de Arthrocnemum macrostachyum (Moric.) K. Koch (Rodríguez Delgado et al., 2000).

Las características ecológicas de ambas asociaciones son diferentes, pues la ecología de la Sarcocornia perennis (Mill.) A.J.Scott que cita Samek (1973) es distinta de la de $S$. perennis de las islas Canarias y de la Península Ibérica, donde esta especie necesita una mayor y constante inundación (Galán de Mera et al., 1997). Según los artículos 31 y 39 del Código es necesario volver a nombrar a la asociación canaria:

\section{Zygophyllo fontanesii-Sarcocornietum perennis (Fernández \& Santos) Galán de Mera, García-Fuentes \& Martínez- Quesada nom. nov. hoc loco \\ [Sarcocornietum perennis Fernández \&} Santos in Lazaroa 5: 148 (1983), holotypus: inv. 4, tab. 2, Orzola, Lanzarote].

Vegetación de los niveles inferiores del litoral sobre suelos arcillosos inundados durante la pleamar. Esta asociación también incluye a la subasociación limonietosum canariensis Fernández \& Santos 1983 que, caracterizada por el endemismo de la isla de Lobos Limonium 
ovalifolium subsp. canariense Pignatti (Suárez García et al., 2004), prefiere los suelos permanentemente inundados en el nivel inferior de la asociación (Fernández \& Santos, 1983; Rodríguez Delgado et al., 2000).

El esquema sintaxonómico donde se incluyen ambas asociaciones quedaría de la siguiente forma:

BATIDO-SARCOCORNIETEA AMBIGUAE Borhidi 1996, nom. mut. Galán de Mera et al. 2009 [Clase neotropical]

+ Batido-Sarcocornietalia ambiguae Borhidi 1996, nom. mut. Martínez-Quesada 2014

* Frimbristylo-Sarcocornion perennis Borhidi 1996, nom. mut. Martínez-Quesada 2014

1. Sarcocornietum perennis Samek 1973, nom. mut. hoc loco

[Asociación de suelos arcillosos inundados periódicamente en contacto con los manglares de la isla de Cuba]

SARCOCORNIETEA FRUTICOSAE Br.-B1. \& Tüxen ex A. \& O. Bolòs 1950, nom. mut. Rivas-Martínez et al. 2002 [Clase europea occidental, mediterránea y macaronésica] + Sarcocornietalia fruticosae Br.-Bl. 1933, nom. mut. Rivas-Martínez et al. 2002

* Sarcocornion fruticosae Br.-Bl. 1933, nom. mut. Rivas-Martínez et al. 2002

** Sarcocornienion perennis Rivas-Martínez in Rivas-Martínez \& Costa 1984, nom. mut. Rivas-Martínez et al. 2002

2. Zygophyllo fontanesii-Sarcocornietum perennis (Fernández \& Santos) Galán de Mera, García-Fuentes \& MartínezQuesada nom. nov. hoc loco

sarcocornietosum perennis

[Comunidad canaria oriental de los niveles inferiores del litoral sobre suelos arcillosos inundados durante la pleamar]

limonietosum canariensis Fernández \& Santos 1983

[Subasociación de la isla de Lobos situada en el nivel inferior de la asociación]

\section{BIBLIOGRAFÍA}

BORHIDI, A. -1996- Phytogeography and vegetation ecology of Cuba. Akadémiai Kiadó, Budapest.

BORHIDI, A., O. MUÑIZ \& E. DEL RISCO -1983Plant communities of Cuba, I. Fresh and salt water, swamp and coastal vegetation. Acta Bot. Hungarica 29(1-4): 337-376.

FERNÁNDEZ, M. \& A. SANTOS -1983- La vegetación del litoral de Canarias, I. Arthrocnemetea. Lazaroa 5: 143-155.

GALÁN DE MERA, A., E. LINARES PEREA, J. CAMPOS DE LA CRUZ \& J.A. VICENTE ORELLANA -2009- Nuevas observaciones sobre la vegetación del sur del Perú. Del Desierto Pacífico al Altiplano. Acta Bot. Malacitana 34: 107-144.

GALÁN DE MERA, A., I. SÁNCHEZ GARCÍA \& J.A. VICENTE ORELLANA -1997- Coastal plant communities of the southwestern Iberian Peninsula, Spain and Portugal. Phytocoenologia 27(3): 313-352.

KNAPP, R. -1964- Höhere Vegetations-Einheiten einiger Gebiete der holarktischen und neotropischen Floren-Reiche. Geobot. Mitteil. 28: 1-11.

MARTÍNEZ-QUESADA, E. -2014- Nuevos sintáxones del archipiélago de los Jardines de la Reina, Cuba. Acta Bot. Malacitana 39: 99-115.

PEINADO, M., F. ALCARAZ \& J. DELGADILLO -1995- Syntaxonomy of some halophilous communities of North and Central America. Phytocoenologia 25(1): 23-31.

RIVAS-MARTÍNEZ, S., T.E. DÍAZ, F. FERNÁNDEZ-GONZÁLEZ, J. IZCO, J. LOIDI, M. LOUSÃ \& A. PENAS -2002- Vascular plant communities of Spain and Portugal. Addenda to the syntaxonomical checklist of 2001. Itinera Geobot. 15(2): 433-922.

RIVAS-MARTÍNEZ, S., F. FERNÁNDEZGONZÁlEZ, J. LOIDI, M. LOUSÃ \& A. PENAS -2001- Syntaxonomical checklist of vascular plant communities of Spain and Portugal to association level. Itinera Geobot. 14: 5-341.

RODRÍGUEZ DELGADO, O., A. GARCÍA 
GALLO \& J.A. REYES BETANCORT -2000Estudio fitosociológico de la vegetación actual de Fuerteventura (islas Canarias). Vieraea 28: 61-98.

SAMEK, V. -1973- Vegetación litoral de la costa norte de la provincia de La Habana. Acad. Ci. Cuba, ser. For. 18: 3-85.

SUÁREZ GARCÍA, C., A. ROCA \& B. VILCHES -2004-Limonium ovalifolium subsp. canariense Pignatti. In: Bañares Baudet, A., G. Blanca, J. Güemes Heras, J.C. Moreno Saiz \& S. Ortiz (eds.) Atlas y Libro Rojo de la flora vascular amenazada de España. Táxones prioritarios: 366-367. Ministerio de Medio Ambiente. Madrid.
WEBER, J.E., J. MORAVEC \& J.P. THEURILLAT -2000- International Code of Phytosociological Nomenclature. 3rd edition. J. Veg. Sci. 11: 739-768.

Dirección de los autores. 'Laboratorio de Botánica, Facultad de Farmacia, Universidad San Pablo-CEU, Apartado 67, E-28660 Boadilla del Monte, Madrid, España. ${ }^{2}$ Departamento de Biología Animal, Biología Vegetal y Ecología, Universidad de Jaén, Campus Las Lagunillas s/n, 23071 Jaén, España. ${ }^{3}$ Centro de Investigaciones del Medio Ambiente de Camagüey, Cisneros $n^{\circ} 105$ (altos)/ Pobre y Ángel, Camagüey 1, 70100, Cuba. *Autor para correspondencia: agalmer@ceu.es. 